\title{
Treadmill exercise in obese maternal rats during pregnancy improves spatial memory through activation of phosphatidylinositol 3-kinase pathway in the hippocampus of rat pups
}

\author{
Young Jun Ko', Bo-Kyun Kim², Eun-Sang Ji3,4,* \\ ${ }^{1}$ Major in Sport Service Practice, College of Welfare Convergence, Kangnam University, Yongin, Korea \\ 2Department of Emergency Technology, College of Health Science, Gachon University, Incheon, Korea \\ ${ }^{3}$ Department of Sport \& Health Care, College of Art \& Culture, Sangmyung University, Seoul, Korea \\ ${ }^{4}$ Department of Physiology, College of Medicine, Kyung Hee University, Seoul, Korea
}

\begin{abstract}
Maternal nutrition is necessary for the growth of the fetus, and excessive intake of nutrients interferes with brain development in offspring. In the current study, the effect of treadmill running during pregnancy in obese maternal rats on spatial learning memory and spatial working memory in rat pups was investigated. Phosphorylation of phosphatidylinositol 3-kinase (PI3K), protein kinase B (Akt), and extracellular signal-regulated kinase 1 and 2 (ERK1/2) was also identified in rat pups. Female rats were divided into the normal diet group and the high-fat diet group for 7 weeks, including pregnancy and lactation. Maternal treadmill running was performed for 4 weeks. The born rat pups were classified into a control group, a treadmill exercise group, a high-fat diet group, a high-fat diet and treadmill exercise group according to the sta-
\end{abstract}

tus of maternal rats. Radial 8-arm maze task for spatial learning memory and Morris water maze task for spatial working memory were done. Western blot analysis was conducted to determine the expressions of PI3K, Akt, ERK1/2. In the current results, maternal treadmill running during pregnancy improved spatial learning memory and spatial working memory in rat pups born to obese maternal rats. This improving effect of memory was due to the enhanced phosphorylation of PI3K, Akt, and ERK $1 / 2$ by treadmill running.

Keywords: Maternal obesity, Pregnancy, Treadmill exercise, PI3K, Rat pups

\section{INTRODUCTION}

Maternal nutrition is necessary for the growth of the fetus, and excessive intake of nutrients interferes with brain development in offspring. Thus, excessive maternal nutrition leads to obesity, hypertension and metabolic disorders in the pups (Chang et al., 2008; Shankar et al., 2008). The hippocampus integrates all information, including short-term, intermediate, and long-term memory (Snyder et al., 2005). Previously, we reported that treadmill running of obese maternal rats improved short-term memory by increasing brain-derived neurotrophic factor expression in the hippocampus of rat pups born to obese maternal rats (Ji et al., 2020). This improving effect of treadmill exercise on short-term memory was attributed to increased cell proliferation in the hippocampus of rat pups born to obese maternal rats (Ji et al., 2020).

Brain-derived neurotrophic factor (BDNF) activates various intracellular signals such as phosphatidylinositol 3-kinase (PI3K), protein kinase B (Akt), and extracellular signal-regulated kinase (ERK) (Numakawa et al., 2010). PI3K is associated with cell growth, proliferation, differentiation, and survival. PI $3 \mathrm{~K}$ is a major factor in the signaling pathway of tyrosine kinase (Huang and Reichardt, 2003), and PI3K produces second messengers such as
${ }^{*}$ Corresponding author: Eun-Sang Ji (iD https://orcid.org/0000-0001-6135-7183 Department of Physiology, College of Medicine, Kyung Hee University,

26 Kyungheedae-ro, Dongdaemun-gu, Seoul 02447, Korea

E-mail: wldmstkd11@hanmail.net

Received: November 2, 2020 / Accepted: December 1, 2020
This is an Open Access article distributed under the terms of the Creative Commons Attribution Non-Commercial License (https://creativecommons.org/licenses/by-nc/4.0/) which permits unrestricted non-commercial use, distribution, and reproduction in any medium, provided the original work is properly cited. 
phosphoinositide-3,4-diphosphate or phosphoinositide-3,4,5-triphosphate (Banadakoppa et al., 2014). Akt is a serine/threonine-specific protein kinase and regulates many downstream effectors such as nuclear factor- $\mathrm{\kappa B}$ and $\mathrm{Bcl}-2$ family proteins (Song et al., 2005). Akt plays critical role in glucose metabolism, apoptosis, cell proliferation, transcription, and cell migration (Numakawa et al., 2010). Activated Akt modulates activation or deactivation the substrate through kinase activity. Mitogen-activated protein kinase (MAPK) is one of the protein kinases and MAPK is specific to serine and threonine. MAPK regulates gene expression, proliferation, differentiation, mitosis, apoptosis, and cell survival (Pearson et al., 2001). ERK includes ERK1, ERK2, ERK5, and ERK7 etc. ERK 1 and 2 (ERK1/2) is called classical MAPK, and is stimulated by proliferation by epithelial growth factor or platelet-derived growth factor, or activated by cell differentiation factors such as nerve growth factor to promote cell proliferation or differentiation.

The purpose of current study was to evaluate the effect of treadmill running during pregnancy in obese maternal rats on spatial learning memory and spatial working memory in rat pups. Phosphorylation of PI3K, Akt, and ERK1/2 was also identified in rat pups to evaluate the mechanism of exercise.

\section{MATERIALS AND METHODS}

\section{Animals}

Four weeks old Sprague-Dawley male rats $(\mathrm{n}=8)$ and female rats $(n=16)$ were used for this experiment. After mating with male rats for 48 hours, female rats were bred separately. Obesity was caused by feeding female rats a high-fat diet ( $60 \%$ fat). Female rats were divided into two groups: normal diet group $(n=8)$ and high-fat diet group $(\mathrm{n}=8)$. The speed of maternal treadmill running was first $3 \mathrm{~m} / \mathrm{min}$ for the $5 \mathrm{~min}$, next $5 \mathrm{~m} / \mathrm{min}$ for $5 \mathrm{~min}$, and lastly $8 \mathrm{~m} / \mathrm{min}$ for $20 \mathrm{~min}$, once $30 \mathrm{~min}, 5$ times a week, during 4 weeks. The born rat pups were classified into four groups according to the conditions of the maternal rats: control group, treadmill exercise group, high-fat diet group, and high-fat diet and treadmill exercise group ( $\mathrm{n}=10$ in each group). The approval number for this experiment was obtained from the Kyung Hee University Institutional Animal Care and Use Committee in Seoul, Korea (KHUASP [SE]-17-099).

\section{Radial 8-arm maze task}

Radial 8-arm maze task was done to detect spatial learning memory as mentioned below (Ko et al., 2019). The radial 8-arm maze devise consisted of a central octagonal plate $30 \mathrm{~cm}$ in diameter and 8 radial arms $50 \mathrm{~cm}$ long and $10 \mathrm{~cm}$ wide, the devise placed $1 \mathrm{~m}$ above the floor. A small container with a diameter of $3 \mathrm{~cm}$ and a depth of $1 \mathrm{~cm}$ was filled with water at the tip of the arm. After training 3 times, the work of the radial 8-arm maze was tested. The rat pups were water inhibited for 24 hours prior working with the radial 8-arm maze task, and explored the water for 5 min during the radial 8-arm maze task. The experiment was ended when rat pups found water in the arms or $5 \mathrm{~min}$ had passed. The number of correct choices was counted as the number before the first error occurred, and re-entry to the previously visited arm was considered as an error.

\section{Morris water maze task}

Morris water maze task was conducted to detect spatial working memory as mentioned below (Park et al., 2019). To do this, rat pups need to know the spatial location of the hidden platform in a black circular pool with a diameter of $150 \mathrm{~cm}$ and a height of $50 \mathrm{~cm}$ filled with clear water $\left(25^{\circ} \mathrm{C} \pm 1^{\circ} \mathrm{C}\right)$. The hidden platform, $15 \mathrm{~cm}$ in diameter and $35 \mathrm{~cm}$ high, disguised itself as transparent on a black background, $2 \mathrm{~cm}$ below the water surface in the center of the northern quadrant. The distal visual cues lie on the walls around the pool, and the position of the cues was not changed through the experiment. All rat pups were trained for 3 consecutive days, 3 times a day prior to the Morris water maze task measurement. Morris water maze task was performed $24 \mathrm{hr}$ after the last training. When finding the platform, rat pups were left on the platform for $30 \mathrm{sec}$, and if rat pups could not find the platform within $60 \mathrm{sec}$, the hand guided them to the platform. The rat pups were provided $60 \mathrm{sec}$ for the Morris water maze task, and all data were automatically measured by Smart Video Tracking System (Smart ver. 2.5, Panlab, Barcelona, Spain).

\section{Tissue preparation}

After Morris water maze task, Zoletil 50 (10 mg/kg, Vibac Laboratories, Carros, France) was injected intraperitoneally to anesthesia, and $50 \mathrm{mM}$ phosphate-buffered saline was injected through heart, and then fixed with $4 \%$ paraformaldehyde in $100 \mathrm{mM}$ phosphate buffer $(\mathrm{pH}, 7.4)$. Brains were excised and the brains were fixed overnight using the same fixative and transferred to a $30 \%$ sucrose solution to prevent freezing. A frozen microtome (Leica, Nussloch, Germany) was used to make $40-\mu \mathrm{m}$-thick sagittal sections.

\section{Western blot analysis}

As mentioned below, western blotting for PI3K, Akt, and 
ERK1/2 was done (Park et al., 2019). Hippocampal tissues were lysed in a lysis buffer comprising $1 \mathrm{mM}$ phenylmethylsulfonyl fluoride, $50 \mathrm{mM}$ Tris- $\mathrm{HCl}(\mathrm{pH}, 7.5), 150 \mathrm{mM} \mathrm{NaCl}, 0.1 \%$ sodium dodecyl sulfate, $0.5 \%$ deoxycholic acid, $1 \%$ Nonidet P40, $100-\mathrm{mg} / \mathrm{mL}$ leupeptin. Rabbit anti-GAPDH antibody (1:1,000; Cell Signaling Technology, Beverly, MA, USA), mouse anti-total PI3K (t-PI3K) antibody (1:1,000; Santa Cruz Biotechnology, CA, USA), rabbit anti-phosphorylated-PI3K antibody (p-PI3K) (1:1,000; Santa Cruz Biotechnology), rabbit anti-total-Akt (t-Akt) antibody (1:1,000; Santa Cruz Biotechnology), rabbit anti-phosphorylated-Akt (p-Akt) antibody (1:1,000; Santa Cruz Biotechnology), rabbit anti-total ERK1/2 (t-ERK1/2) antibody (1:1,000; Cell Signaling Technology), rabbit anti-phosphorylated-ERK1/2 (p-ERK1/2) antibody (1:1,000; Cell Signaling Technology) were used as the primary antibody. Horseradish peroxidase-conjugated secondary antibodies were treated after washing. Image-Pro Plus computer-assisted image analysis system (Media Cyberbetics Inc., Silver Spring, MD, USA) was used for the quantification of the bands.

\section{Data analysis}

Data was analyzed by IBM SPSS Statistics ver. 21.0 (IBM Co., Armonk, NY, USA), and one-way analysis of variance followed Duncan post boc test compared among the groups. The results were expressed as the mean \pm standard error of the mean with $P<0.05$ considered as statistically significant.

\section{RESULTS}

\section{Spatial learning ability}

For the effect of treadmill running during pregnancy of obese maternal rats on the spatial learning ability of rat pups, the radial 8-arm maze task was conducted (Fig. 1). In the high-fat diet group, the time taken to find all the water in 8 mazes was increased compared to the control group $(P<0.05)$. Treadmill running reduced the time taken to find all the water in the high-fat diet group $(P<$ 0.05). In the high-fat diet group, the number of correct choices that found water in succession was reduced compared to the control group $(P<0.05)$. Treadmill running increased the number of correct choices to water continuously in the high-fat diet group $(P<0.05)$. In the high-fat diet group, the number of errors entering the maze was increased compared to the control group $(P<$ $0.05)$. Treadmill running reduced the number of errors in the high-fat diet group $(P<0.05)$.

\section{Spatial working memory}

For the effect of treadmill running during pregnancy of obese maternal rats on the spatial working memory of rat pups, the Morris water maze task was conducted (Fig. 2). In the high-fat diet group, the time spent around probe quadrant was decreased compared to the control group $(P<0.05)$. Treadmill running increased the time spent around probe quadrant in the high-fat diet group $(P<0.05)$.

\section{PI3K expression}

For the effect of treadmill running during pregnancy of obese maternal rats on the PI $3 \mathrm{~K}$ expression in the hippocampus of rat pups, PI3K expression was determined (Fig. 3). The ratio of $\mathrm{p}-\mathrm{PI} 3 \mathrm{~K} / \mathrm{t}-\mathrm{PI} 3 \mathrm{~K}$ was decreased in the high-fat diet group compared to the control group $(P<0.05)$. Treadmill running increased the ratio of $\mathrm{p}-\mathrm{PI} 3 \mathrm{~K} / \mathrm{t}-\mathrm{PI} 3 \mathrm{~K}$ in the high-fat diet group $(P<0.05)$.

\section{Akt expression}

For the effect of treadmill running during pregnancy of obese
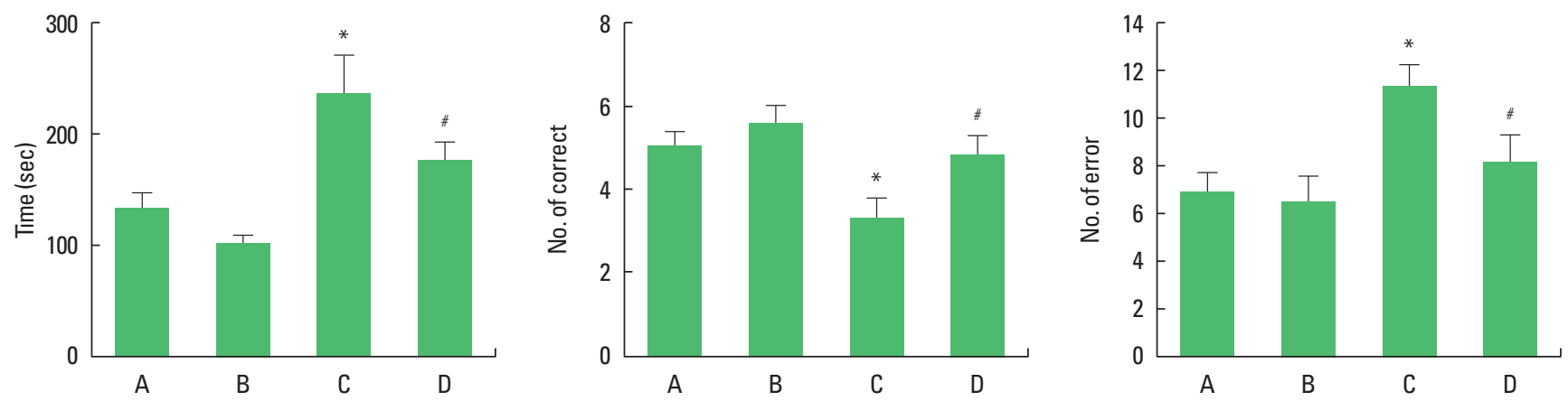

Fig. 1. Spatial learning ability of rat pups. Left panel: time spent for seeking water. Middle panel: number of correct choices. Right panel: number of errors. A, control group; $B$, treadmill exercise group; $C$, high-fat diet group; $D$, high-fat diet and treadmill exercise group. ${ }^{*} P<0.05$ compared to the control group. ${ }^{\sharp} P<0.05$ compared to the high-fat diet group. Data means the mean \pm standard error of the mean. 
A

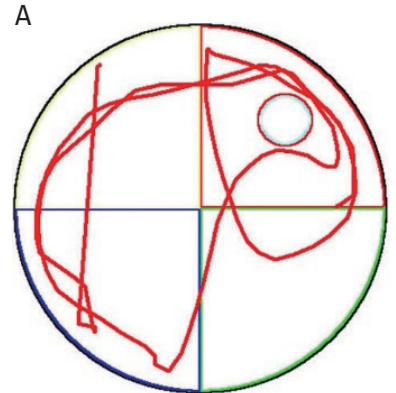

B
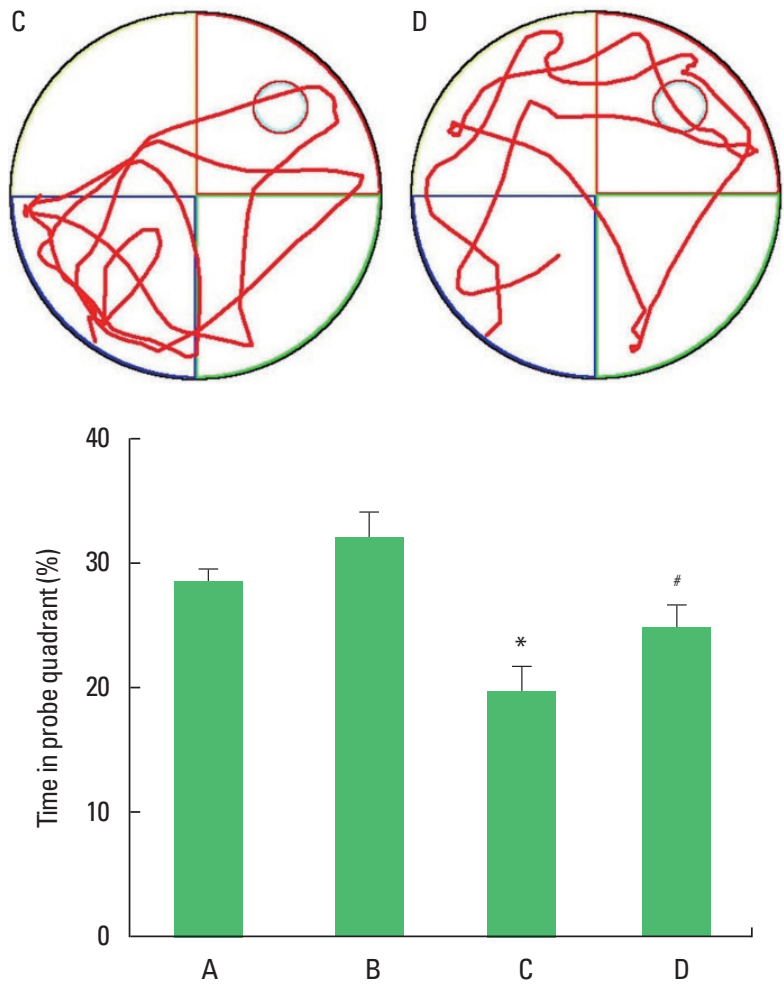

Fig. 2. Spatial working memory of rat pups. Upper panel: Morris water maze task. Lower panel: time spent around probe quadrant in each group. A, control group; $B$, treadmill exercise group; $C$, high-fat diet group; D, high-fat diet and treadmill exercise group. ${ }^{*} P<0.05$ compared to the control group. ${ }^{\#} P<0.05$ compared to the high-fat diet group. Data means the mean \pm standard error of the mean.

maternal rats on the Akt expression in the hippocampus of rat pups, Akt expression was determined (Fig. 4). The ratio of p-Akt/ $\mathrm{t}$-Akt was decreased in the high-fat diet group compared to the control group $(P<0.05)$. Treadmill running increased the ratio p-Akt/t-Akt in the high-fat diet group $(P<0.05)$.

\section{ERK1/2 expression}

For the effect of treadmill running during pregnancy of obese maternal rats on the ERK1/2 expression in the hippocampus of rat pups, ERK1/2 expression was determined (Fig. 5). The ratio
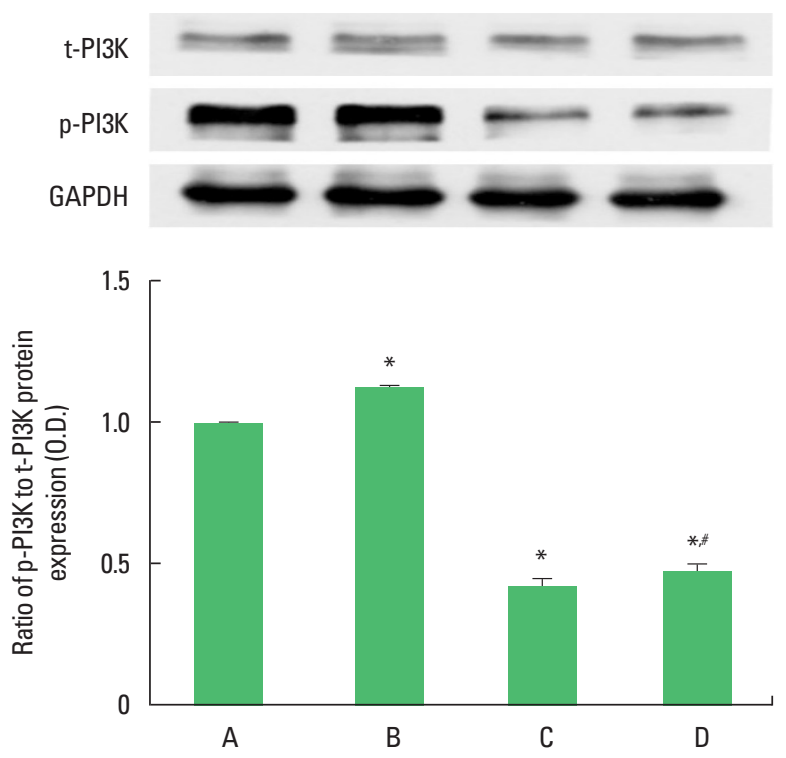

Fig. 3. Phosphatidylinositol 3-kinase (PI3K) expression in the hippocampus of rat pups. Upper panel: western blot analysis of total-PI3K (t-PI3K) and phosphorylated (p-PI3K) in the hippocampus. Lower panel: p-PI3K/t-PI3K ratio in each group. $A$, control group; $B$, treadmill exercise group; $C$, high-fat diet group; $D$, high-fat diet and treadmill exercise group. ${ }^{*} P<0.05$ compared to the control group. ${ }^{\sharp} P<0.05$ compared to the high-fat diet group. Data means the mean \pm standard error of the mean.
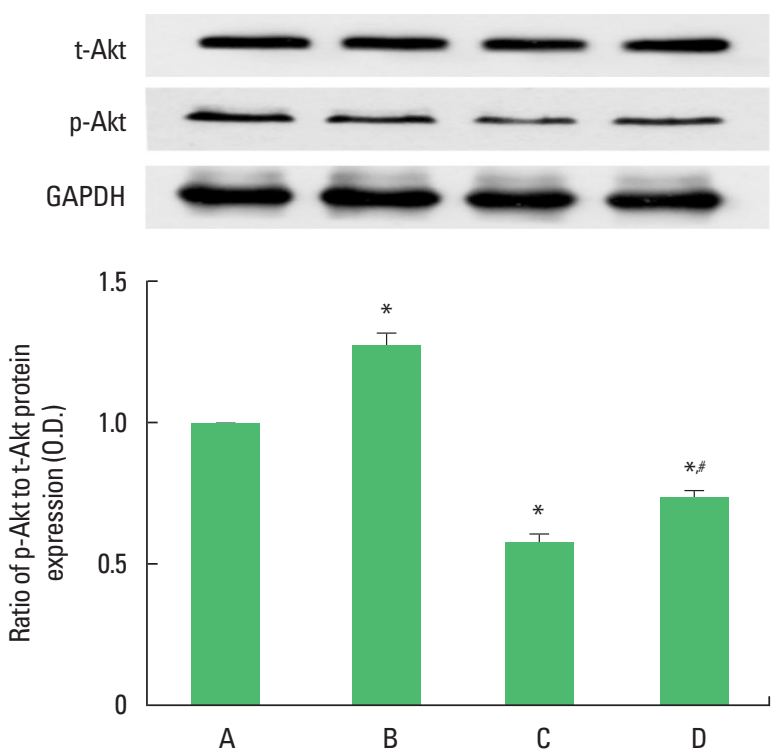

Fig. 4. Protein kinase B (Akt) expression in the hippocampus of rat pups. Upper panel: western blot analysis of total-Akt (t-Akt) and phosphorylated (p-Akt) in the hippocampus. Lower panel: p-Akt/t-Akt ratio in each group. A, control group; $B$, treadmill exercise group; $C$, high-fat diet group; D, high-fat diet and treadmill exercise group. ${ }^{*} P<0.05$ compared to the control group. ${ }^{*} P<0.05$ compared to the high-fat diet group. Data means the mean \pm standard error of the mean. 

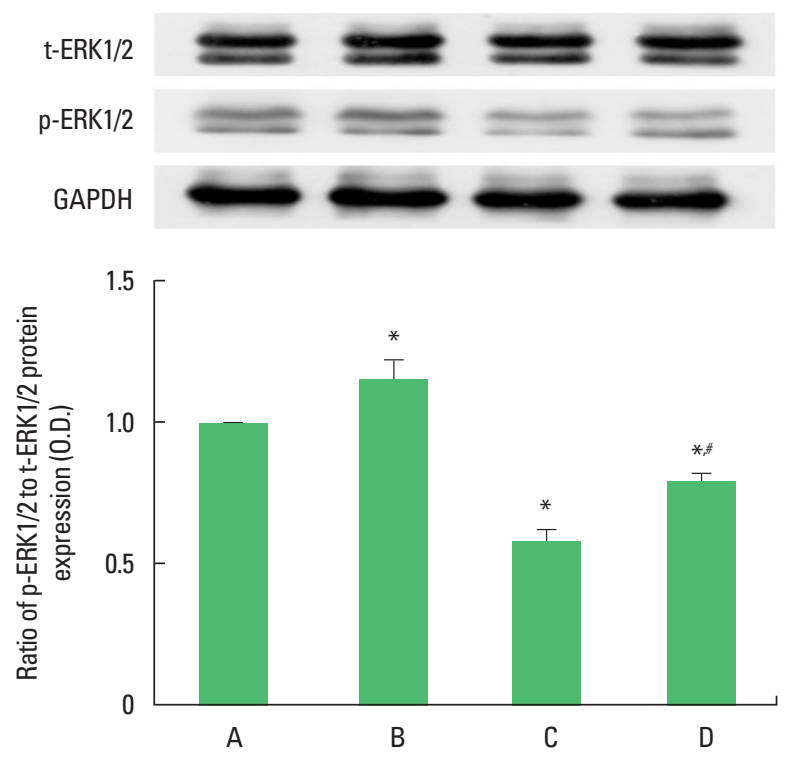

Fig. 5. Extracellular signal-regulated kinase 1 and 2 (ERK1/2) expression in the hippocampus of rat pups. Upper panel: western blot analysis of total-ERK1/2 (t-ERK1/2) and phosphorylated (p-ERK1/2) in the hippocampus. Lower panel: p-ERK1/2/t-ERK1/2 ratio in each group. $A$, control group; $B$, treadmill exercise group; $C$, high-fat diet group; $D$, high-fat diet and treadmill exercise group. ${ }^{*} P<0.05$ compared to the control group. ${ }^{\#} P<0.05$ compared to the high-fat diet group. Data means the mean \pm standard error of the mean.

of p-ERK1/2/t-ERK1/2 was decreased in the high-fat diet group compared to the control group $(P<0.05)$. Treadmill running increased the ratio $\mathrm{p}-\mathrm{ERK} 1 / 2 / \mathrm{t}-\mathrm{ERK} 1 / 2$ in the high-fat diet group $(P<0.05)$.

\section{DISCUSSION}

Obesity causes systemic inflammatory conditions and is also associated with cognitive impairment (Bilbo and Tsang, 2010). Maternal swimming during pregnancy increased short-term memory through enhancing cell proliferation in the hippocampus of rat pups (Lee et al., 2006). It is well known that exercise of the maternal rats increased memory in offspring (Akhavan et al., 2008; Kim et al., 2007). In the current results, spatial learning memory and spatial working memory were decreased in rat pups born to maternal rats with a high-fat diet compared to control maternal rats. Treadmill running of the maternal rats significantly improved spatial learning memory and spatial working memory in rat pups born to maternal rats with a high-fat diet.

Protein phosphorylation by the tyrosine kinase B receptor (TrkB) induces the activation of $\mathrm{PI} 3 \mathrm{~K}$ and $\mathrm{Akt}$, regulating the survival of neurons and inducing the activity of ERK, thereby activating the transcription factor cyclic AMP-response element binding (Kaplan and Miller, 2000). PI3K and ERK activation is mediated by protein binding to phosphorylated TrkB receptor (Numakawa et al., 2010). The MAPK signaling system is a factor that regulates embryogenesis, cell differentiation, proliferation, and death, and ERK1/2 is an important kinase that determines cell fate in the MAPK signaling system. ERK is essential for cell survival by activating $\mathrm{PI} 3 \mathrm{~K}$ and Akt signaling pathways and promoting cell differentiation and growth (Santos et al., 2010). ERK is essential for cell survival by activating PI3K and Akt signaling pathways to promote cell differentiation and growth (Santos et al., 2010). In the current results, phosphorylation of PI3K, Akt, and ERK1/2 in rat pups born to maternal rats with a high-fat diet was decreased compared to the control group. This means maternal rats with high-fat diet caused dephosphorylation of PI3K, Akt, and ERK1/2 in rat pups.

In a previous study, maternal treadmill running during pregnancy improved short-term memory in rat pups born to obese maternal rats (Ji et al., 2020). The improving effect of short-term memory was achieved by enhancing neurogenesis through increased expression of BDNF and TrkB by treadmill running (Ji et al., 2020). In the current results, phosphorylation of PI3K, Akt, and ERK1/2, which are involved in neuronal birth and survival in the hippocampus, was significantly enhanced in rat pups by treadmill running of maternal rats with high-fat diet group. This indicates treadmill running in maternal rats with high-fat diet enhanced phosphorylation of PI3K, Akt, and ERK1/2 in rat pups.

In the current study, maternal treadmill exercise during pregnancy improved spatial learning memory and spatial working memory in rat pups born to obese maternal rats. This increasing effect was due to the enhanced phosphorylation of PI3K, Akt, and ERK1/2 in rat pups by treadmill running.

\section{CONFLICT OF INTEREST}

No potential conflict of interest relevant to this article was reported.

\section{REFERENCES}

Akhavan MM, Emami-Abarghoie M, Safari M, Sadighi-Moghaddam B, Vafaei AA, Bandegi AR, Rashidy-Pour A. Serotonergic and noradrenergic lesions suppress the enhancing effect of maternal exercise during pregnancy on learning and memory in rat pups. Neuroscience 2008; 15:1173-1183. 
Banadakoppa M, Goluszko P, Liebenthal D, Nowicki BJ, Nowicki S, Yallampalli C. PI3K/Akt pathway restricts epithelial adhesion of $\mathrm{Dr}^{+}$Escherichia coli by down-regulating the expression of decay accelerating factor. Exp Biol Med (Maywood) 2014;239:581-594.

Bilbo SD, Tsang V. Enduring consequences of maternal obesity for brain inflammation and behavior of offspring. FASEB J 2010;24:2104-2115.

Chang GQ, Gaysinskaya V, Karatayev O, Leibowitz SF. Maternal high-fat diet and fetal programming: increased proliferation of hypothalamic peptide-producing neurons that increase risk for overeating and obesity. J Neurosci 2008;28:12107-12119.

Huang EJ, Reichardt LF. Trk receptors: roles in neuronal signal transduction. Annu Rev Biochem 2003;72:609-642.

Ji ES, Kim YM, Ko YJ, Baek SS. Treadmill exercise in obese maternal rats during pregnancy improves short-term memory through neurogenesis in the hippocampus of rat pups. J Exerc Rehabil 2020;16:392-397.

Kaplan DR, Miller FD. Neurotrophin signal transduction in the nervous system. Curr Opin Neurobiol 2000;10:381-391.

Kim H, Lee SH, Kim SS, Yoo JH, Kim CJ. The influence of maternal treadmill running during pregnancy on short-term memory and hippocampal cell survival in rat pups. Int J Dev Neurosci 2007;25:243-249.

Ko IG, Kim CJ, Kim H. Treadmill exercise improves memory by up-regulating dopamine and down-regulating D2 dopamine receptor in traumatic brain injury rats. J Exerc Rehabil 2019;15:504-511.
Lee HH, Kim H, Lee JW, Kim YS, Yang HY, Chang HK, Lee TH, Shin MC, Lee MH, Shin MS, Park S, Baek S, Kim CJ. Maternal swimming during pregnancy enhances short-term memory and neurogenesis in the hippocampus of rat pups. Brain Dev 2006;28:147-154.

Numakawa T, Yokomaku D, Richards M, Hori H, Adachi N, Kunugi H. Functional interactions between steroid hormones and neurotrophin BDNF. World J Biol Chem 2010;1:133-143.

Park SS, Shin MS, Park HS, Kim TW, Kim CJ, Lim BV. Treadmill exercise ameliorates nicotine withdrawal-induced symptoms. J Exerc Rehabil 2019;15:383-391.

Pearson G, Robinson F, Beers Gibson T, Xu BE, Karandikar M, Berman K, Cobb MH. Mitogen-activated protein (MAP) kinase pathways: regulation and physiological functions. Endocr Rev 2001;22:153-183.

Santos AR, Comprido D, Duarte CB. Regulation of local translation at the synapse by BDNF. ProgNeurobiol 2010;92:505-516.

Shankar K, Harrell A, Liu X, Gilchrist JM, Ronis MJ, Badger TM. Maternal obesity at conception programs obesity in the offspring. Am J Physiol Regul Integr Comp Physiol 2008;294:528-538.

Snyder JS, Hong NS, McDonald RJ, Wojtowicz JM. A role for adult neurogenesis in spatial long-term memory. Neuroscience 2005;130:843852.

Song G, Ouyang G, Bao S. The activation of Akt/PKB signaling pathway and cell survival. J Cell Mol Med 2005;9:59-71. 